\title{
BEDAUERLICH, ABER UNABWENDBAR: WELTKONGRESS AURIKULOMEDIZIN ABGESAGT
}

\section{Liebe Kolleginnen und Kollegen,}

die Zeit vergeht wie im Flug. Seit nunmehr 15 Monaten ist der neue Vorstand im Amt. Es ist nicht zuletzt unserem langjährigen Vorsitzenden Herrn Bahr und seiner Frau zu verdanken, dass der Übergang so gut gelungen ist. Aus diesem Grunde sei nochmals ein herzliches Dankeschön für die Begleitung im vergangenen Jahr ausgesprochen.

Der neueVorstand hat sich die Handlungsmaxime gesetzt: „Der Tradition verpflichtet - neue Schritte wagen“. Diese Grundeinstellung hat das vergangene Jahr geprägt und wird auch in den kommenden Jahren bedeutsam bleiben. Der neu angebotene Kurs zur Liquidakupunktur wurde gut angenommen. In regelmäßigen Abständen erreichen mich Rückmeldungen von begeisterten Kollegen. Der Tenor ist immer ähnlich, daher sei eine Nachricht auszugsweise wiedergegeben: ,... Ich bin Zahnarzt und habe bereits mehrere Kurse zur Ohrakupunktur besucht. Allerdings habe ich es nie geschafft, die Ohrakupunktur im Rahmen der zahnärztlichen Behandlung intensiv anzuwenden, obwohl ich nach wie vor davon begeistert bin. Anders war es nun bei der Liquidakupunktur. Seit Ihrem Kurs habe ich etwa 12-15 Patienten ... behandelt. Die Resonanz ist bisher überwältigend. ... Alle, ..., waren von der schnellen und zumeist nachhaltigen Wirkung verblüfft. Ihnen und Ihren Kollegen, die dies lehren, ein herzliches Dankeschön!“(Dr. F. Mortazavi; E-Mail vom 05.02.2016)

Damit kommen wir zu den Neuerungen für das Jahr 2016. Während die Liquidakupunktur eine einfache und sichere Behandlungsmethode ist, so ist die klassische Ohrakupunktur, Körperakupunktur und Störherddiagnostik für komplexere Fälle unverzichtbar. Auch hier wollen wir Ihnen eine Hilfe an die Hand geben, damit der am häufigsten genannte Hinderungsgrund für die Anwendung von Akupunktur in der Alltagspraxis nahezu entfällt:
DER ORGANISATIONSAUFWAND. Aus diesem Grunde wird es im Herbst an verschiedenen Kursorten in Deutschland einen Arzthelferinnenkurs zur Akupunktur geben. Damit schließen wir die Lücke vom „Akupunkturbefähigten Arzt“ zum ,leidenden Patienten“. Die Arzthelferin soll den Arzt/die Ärztin sowohl in der Patientenkommunikation unterstützen, als auch die Organisation der Termine und Räume perfekt an die Bedürfnisse einer Akupunkturpraxis anpassen können. Abrechnungswesen und Qualitätsmanagement gehören ebenso in die Ausbildung wie auch die Möglichkeiten und Grenzen delegierbarer Leistungen. Schauen Sie mal wieder auf unsere Seminarseite (http://www.akupunktur-seminare.de). Dort finden Sie das ausführliche Kursprogramm zum Arzthelferinnenkurs.

Manchmal kommt es anders als geplant: Obwohl die Organisation des WCAM Kongresses 2016 in Wien bereits auf gutem Wege war, musste dieser abgesagt werden, da zwei besonders wichtige Referenten des Weltkongresses für Aurikulomedizin erkrankt sind und eine zugesagte hohe Forschungsunterstützung über den mehrfachen fMRT-Nachweis von Ohrakupunkturpunkten in ihrer Korrespondenz zu Gehirnarealen dann doch nicht gewährt wurde. So müssen wir das Thema Aurikulomedizin auf das Jahr 2017 verlegen, der Weltkongress wird dann in Singapur stattfinden (www.auriculotherapysymposium.com).

Mit guten Wünschen für die kommende Zeit und viel Freude an unserem Kursangebot!

Ihr

B. Ramme

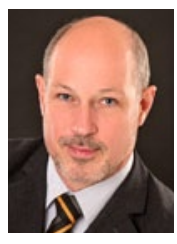

Dr. med. Bernd Ramme

1. Vorsitzender der DAA e.V.,

Osserstraße 40, D-81679 München

Tel. $+4989 / 8145252$

E-Mail des allg.Büros: kontakt@akupunktur.de, Internet:www.akupunktur.de 